\title{
ATRIBUCIONES DEL ALUMNADO DE MATEMÁTICAS Y MANIFESTACIONES DE ANSIEDAD EN LA ESCUELA
}

\author{
Aitana Fernández-Sogorb \\ Universidad de Alicante \\ aitana.fernandez@ua.es \\ Ricardo Sanmartín López \\ Universidad de Alicante \\ ricardo.sanmartin@ua.es \\ Nancy Cargua García \\ Universidad Central del Ecuador \\ ncarguag@yahoo.es \\ Carlos Edisson Jiménez Ayala \\ Universidad Central del Ecuador \\ cjimeneza@hotmail.com
}

Recepción Artículo: 27 octubre 2021

Admisión Evaluación: 27 octubre 2021

Informe Evaluador 1: 28 octubre 2021

Informe Evaluador 2: 29 octubre 2021

Aprobación Publicación: 30 octubre 2021

\section{RESUMEN}

En el área de matemáticas el alumnado puede atribuir sus resultados académicos de éxito y fracaso a múltiples causas. Según las dimensiones causales que tengan estas autoatribuciones, la probabilidad de que los estudiantes experimenten ansiedad cognitiva, conductual y psicofisiológica puede aumentar o disminuir. A pesar de la importancia de estudiar estos constructos para comprender mejor el comportamiento de los alumnos, pocos trabajos han abordado su relación en la etapa de la adolescencia. La presente investigación tuvo como objetivo analizar la capacidad predictiva de las autoatribuciones causales en matemáticas sobre la ansiedad cognitiva, la ansiedad conductual y la ansiedad psicofisiológica. Se reclutó una muestra de 432 adolescentes con edades comprendidas entre los 12 y los 18 años $(M=14.74 ; D E=1.69)$. Los participantes respondieron al Inventario de Ansiedad Escolar (IAES) y a la Sydney Attribution Scale (SAS) de forma voluntaria y anónima. Diversos modelos de regresión logística informaron del valor de odd ratio (OR) superior a 1 para la autoatribución del fracaso en matemáticas al esfuerzo y a la capacidad en las tres respuestas de ansiedad examinadas. El valor de OR fue inferior a 1 para el resto de autoatribuciones causales de éxitos y fracasos en ansiedad cognitiva, conductual y psicofisiológica. Estos resultados evidencian que conforme se incrementan las puntuaciones en autoatribuciones causales de éxitos y fracasos en matemáticas a causas externas, la probabilidad de manifestar altos niveles de ansiedad cognitiva, ansiedad conductual y ansiedad psicofisiológica es menor. Por el contrario, conforme aumentan las puntuaciones en las autoatribuciones causales de los fracasos en matemáticas a causas internas, la pro- 


\section{ATRIBUCIONES DEL ALUMNADO DE MATEMÁTICAS Y MANIFESTACIONES DE ANSIEDAD EN LA ESCUELA}

babilidad de experimentar altos niveles de ansiedad cognitiva, conductual y psicofisiológica es mayor. Como consecuencia, para prevenir la ansiedad resulta necesario intervenir en el tipo de autoatribuciones causales escogidas por el alumnado.

Palabras clave: autoatribuciones causales; matemáticas; respuestas de ansiedad; regresión logística

\section{ABSTRACT}

Cognitive, behavioral, and psychophysiological anxiety according to causal self-attributions in mathematics during adolescence. In the area of mathematics, students can attribute their academic results of success and failure to multiple causes. Depending on the causal dimensions of these self-attributions, the probability that students experience cognitive, behavioral, and psychophysiological anxiety may increase or decrease. Despite the importance of studying these constructs to better understand students' behavior, few studies have addressed their relationship in adolescence. The present research aimed to analyze the predictive capacity of causal self-attributions in mathematics on cognitive anxiety, behavioral anxiety, and psychophysiological anxiety. A sample of 432 adolescents aged between 12 and 18 years $(M=14.74 ; S D=1.69)$ was recruited. The participants answered to the School Anxiety Inventory (SAI) and the Sydney Attribution Scale (SAS) voluntarily and anonymously. Various logistic regression models reported an odd ratio (OR) value greater than 1 for the self-attribution of failure in mathematics to effort and ability in the three anxiety responses examined. The OR value was less than 1 for the rest of the causal self-attributions of successes and failures in cognitive, behavioral, and psychophysiological anxiety. These results show that as the scores in causal self-attributions of successes and failures in mathematics to external causes increase, the probability of manifesting high levels of cognitive anxiety, behavioral anxiety and psychophysiological anxiety is lower. On the contrary, as the scores in causal self-attributions of failures in mathematics to internal causes increase, the probability of experiencing high levels of cognitive, behavioral, and psychophysiological anxiety is greater. Consequently, to prevent anxiety, it is necessary to intervene in the type of causal self-attributions chosen by students.

Keywords: causal self-attributions; mathematics; anxiety responses; logistic regression

\section{INTRODUCCIÓN}

La ansiedad en el ámbito escolar debe detectarse de forma temprana al poder derivar en problemas del comportamiento y de asistencia a la escuela (Fernández-Sogorb et al., 2018, 2020a). Asimismo, la teoría de Weiner establece que las atribuciones que los estudiantes realizan sobre sus resultados escolares pueden generar determinados sentimientos (culpa, vergüenza) que, a su vez, influyen positiva o negativamente en la visión que el estudiante tiene de sí mismo (Martinko y Mackey, 2019).

Los estudios sobre las causas que los adolescentes tienden a utilizar para explicar sus éxitos y fracasos en el ámbito académico han constatado la existencia de diferencias significativas en función del sexo, tanto en población extranjera como española. En este sentido, Stipek y Gralinski (1991) analizaron las atribuciones causales de 473 estudiantes norteamericanos de $3^{0}$ y $8^{\circ}$ grado, evaluando su desempeño tras la realización de un examen de matemáticas y cuáles eran las causas implicadas. Estos autores constataron que los chicos, en comparación con sus compañeras, tendían a atribuir en mayor medida su éxito a la capacidad y su fracaso a la mala suerte. Sin embargo, las chicas atribuyeron en mayor medida su fracaso a su falta de capacidad.

Los resultados hallados por Ferreira et al. (2002) en una muestra de 1.594 estudiantes sudamericanos de 14 a 20 años, coinciden con los encontrados en población adolescente de habla inglesa en cuanto a las atribuciones de éxito. Así, estos autores señalaron que las chicas atribuyeron sus éxitos más frecuentemente al esfuerzo, mientras los chicos realizaron atribuciones más relacionadas con la capacidad. Sin embargo, no se encontraron diferencias en función del sexo en las atribuciones causales sobre situaciones de fracaso, siendo la causa principal de este resultado la falta de esfuerzo, tanto para los chicos como para las chicas. En la población española, Cerezo y Casanova (2004), en una muestra de 521 estudiantes de 14 a 18 años, no encontraron diferencias significativas entre chicos y chicas en las atribuciones causales en situaciones de éxito académico. 


\section{OBJETIVOS DE LA INVESTIGACIÓN}

Considerando los estudios anteriormente revisados, esta investigación tuvo como objetivo examinar la capacidad predictiva de las autoatribuciones causales en matemáticas sobre la ansiedad cognitiva, conductual y psicofisiológica en estudiantes adolescentes. Se esperaba que al incrementarse las puntuaciones atribucionales de éxito a la capacidad y al esfuerzo disminuyera la probabilidad de presentar altos niveles en las tres respuestas de ansiedad (hipótesis 1); que al incrementarse las puntuaciones atribucionales de fracaso a la capacidad y al esfuerzo aumentara la probabilidad de presentar altos niveles en las tres respuestas de ansiedad (hipótesis 2); y que conforme se incrementaran las puntuaciones atribucionales de éxito y fracaso a causas externas disminuyera la probabilidad de manifestar altos niveles de ansiedad cognitiva, conductual y psicofisiológica (hipótesis 3).

\section{PARTICIPANTES}

Se reclutaron 432 estudiantes con edades comprendidas entre los 12 y los 18 años $(M=14.74 ; D E=1.69)$, que cursaban estudios desde octavo hasta tercero de bachillerato en ocho centros. La selección de los centros participantes se llevó a cabo a través de un muestreo por conglomerados en la ciudad de Quito.

\section{MÉTODO}

\section{Instrumentos}

Inventario de Ansiedad Escolar (IAES; García-Fernández et al., 2011).

Los análisis psicométricos del IAES indicaron la existencia de 4 factores correlacionados (Ansiedad ante el fracaso y castigo escolar, Ansiedad ante la evaluación social, Ansiedad ante la agresión y Ansiedad ante la evaluación escolar) y tres factores de respuesta independientes (Ansiedad cognitiva, Ansiedad psicofisiológica y Ansiedad conductual). El análisis de fiabilidad de los tres sistemas de respuesta reveló coeficientes de consistencia interna adecuados; entre 0.97 (Ansiedad cognitiva y Ansiedad conductual) y 0.98 (Ansiedad psicofisiológica), y los coeficientes de fiabilidad test-retest, para un intervalo de 4 semanas, variaron de 0.79 (Ansiedad psicofisiológica) a 0.82 (Ansiedad conductual).

Sydney Attribution Scale (SAS; Marsh, 1984).

La SAS incluye 24 supuestos correspondientes a dos áreas académicas (matemática, verbal), dos resultados hipotéticos (éxito 0 fracaso) y tres tipos de causas implicadas (habilidad, esfuerzo, causas externas), la combinación de los cuales genera un total de 72 ítems distribuidos en 12 escalas. En su adaptación a población española se han obtenido datos que informan de su aceptable fiabilidad, validez de constructo y predictiva del rendimiento académico en alumnos de Educación Primaria y Secundaria (Núñez y González-Pineda, 1994; Redondo y Inglés, 2008).

\section{Procedimiento}

Se realizó una reunión con los directores de los centros participantes y con los padres o tutores legales de los estudiantes para solicitar su consentimiento. Acto seguido se administraron los cuestionarios de forma voluntaria y anónima.

\section{Análisis de datos}

Se utilizó la técnica de regresión logística con el procedimiento de pasos hacia adelante basado en el estadístico de Wald. Además, se recurrió al estadístico odd ratio (OR), siguiendo el criterio de De Maris (2003): OR $>1$ implica predicción posotiva, $\mathrm{OR}<1$ supone predicción negativa y $\mathrm{OR}=1$ indica ausencia de predicción.

Los análisis se realizaron con el programa informático SPSS/PC 24.0. 


\section{ATRIBUCIONES DEL ALUMNADO DE MATEMÁTICAS Y MANIFESTACIONES DE ANSIEDAD EN LA ESCUELA}

\section{RESULTADOS ALCANZADOS}

\section{Regresión logística binaria para la probabilidad de presentar alta ansiedad cognitiva en función}

de las atribuciones causales en matemáticas

Los modelos resultantes (véase la Tabla 1) permiten hacer una estimación correcta del $59.6 \%$ de los casos $\left(\chi^{2}=37.72 ; p=<.05\right)$ para la variable MEC= Éxito en matemáticas atribuido a la capacidad; de un $57.9 \%$ de los casos $\left(\chi^{2}=13.58 ; p=<.05\right)$ correspondiente a la variable MEEs= Éxito en matemáticas atribuido al esfuerzo; de un $55.4 \%$ de los casos $\left(\chi^{2}=11.16 ; p=<.05\right)$ correspondiente a la variable MEEX Éxito en matemáticas atribuido a causas externas; de un $64.2 \%$ de los casos $\left(\chi^{2}=78.60 ; p=<.05\right)$ correspondiente a la variable MFC= Fracaso en matemáticas atribuido a la capacidad; de $59 \%$ de los casos $\left(\chi^{2}=26.11 ; p=<.05\right)$ para la variable MFEs= Fracaso en matemáticas atribuido al esfuerzo y de un $57.6 \%$ de los casos $\left(\chi^{2}=15.06 ; p=<.05\right)$ para la variable MFEX = Fracaso en matemáticas atribuido a causas externas. El valor de ajuste de los modelos ( $R^{2}$ Nagelkerke) se situó entre .25 para el modelo MFEx= Fracaso en matemáticas atribuido a causas externas y de .02 para las variables MEEs y MEEX.

Los valores de la odd ratio (OR) fueron superiores a 1 indicando, por tanto, que a medida que aumenta la puntuación en las variables de Atribuciones Causales en Matemáticas aumenta la posibilidad de presentar altas puntuaciones en ansiedad Cognitiva, obteniendo valores en MFC= Fracaso en matemáticas atribuido a la capacidad de 1.99 y en MFEs= Fracaso en matemáticas atribuido al esfuerzo de 1.74 veces mayor por cada punto que aumentan las puntuaciones respectivamente en las dimensiones de atribuciones causales en el área de Matemática. A su vez, también se obtuvo valores de la $O R$ inferiores a 1, por lo que la probabilidad de presentar alta ansiedad cognitiva fue menor, concretamente de .67 para MEC= Éxito en matemáticas atribuido a la capacidad, de .73 para MEEs= Éxito en matemáticas atribuido al esfuerzo, de .74 para MEEx = Matemáticas Éxito Externas, y de .67 para MFEx= Fracaso en matemáticas atribuido a causas externas, por cada punto que se incrementaban las puntuaciones en dichas variables. 
Tabla 1

Regresión logística binaria para la probabilidad de presentar alta ansiedad cognitiva en función de las atribuciones en matemáticas

\begin{tabular}{|c|c|c|c|c|c|c|c|c|c|}
\hline Variable & & $\chi^{2}$ & $\mathrm{R}^{2}$ & $\mathrm{~B}$ & E.T. & Wald & $P$ & $O R$ & I.C. $95 \%$ \\
\hline \multirow[t]{2}{*}{ MEC } & $\begin{array}{l}\text { Clasificados } \\
\text { correctamente: } \\
59.6 \%\end{array}$ & 37.72 & .06 & -.39 & .06 & $\begin{array}{r}35.6 \\
6\end{array}$ & $\begin{array}{r}.00 \\
0\end{array}$ & .67 & \multirow[t]{2}{*}{$.59-.76$} \\
\hline & Constante & & & 1.09 & .17 & $\begin{array}{r}38.0 \\
4\end{array}$ & $\begin{array}{r}.00 \\
0\end{array}$ & 2.98 & \\
\hline \multirow[t]{2}{*}{ MEEs } & $\begin{array}{l}\text { Clasificados } \\
\text { correctamente: } \\
57.9 \%\end{array}$ & 13.58 & .02 & -.31 & .08 & $\begin{array}{r}13.2 \\
3\end{array}$ & $\begin{array}{r}.00 \\
0\end{array}$ & .73 & \multirow[t]{2}{*}{$.61-.86$} \\
\hline & Constante & & & .95 & .23 & $\begin{array}{r}16.3 \\
6\end{array}$ & $\begin{array}{r}.00 \\
0\end{array}$ & 2.60 & \\
\hline \multirow[t]{2}{*}{ MEEx } & $\begin{array}{l}\text { Clasificados } \\
\text { correctamente: } \\
55.4 \%\end{array}$ & 11.16 & .02 & -.29 & .08 & $\begin{array}{r}10.9 \\
6\end{array}$ & $\begin{array}{r}.00 \\
1\end{array}$ & .74 & \multirow[t]{2}{*}{$.63-.88$} \\
\hline & Constante & & & 1.29 & .35 & $\begin{array}{r}13.1 \\
9\end{array}$ & $\begin{array}{r}.00 \\
0\end{array}$ & 3.66 & \\
\hline \multirow[t]{2}{*}{ MFC } & $\begin{array}{l}\text { Clasificados } \\
\text { correctamente: } \\
64.2 \%\end{array}$ & 78.60 & .13 & .68 & .08 & $\begin{array}{r}69.4 \\
5\end{array}$ & $\begin{array}{r}.00 \\
0\end{array}$ & 1.99 & \multirow[t]{2}{*}{$\begin{array}{r}1.69- \\
2.33\end{array}$} \\
\hline & Constante & & & -.95 & .14 & $\begin{array}{r}41.2 \\
3\end{array}$ & $\begin{array}{r}.00 \\
0\end{array}$ & .38 & \\
\hline \multirow[t]{2}{*}{ MFEs } & $\begin{array}{l}\text { Clasificados } \\
\text { correctamente: } \\
59 \%\end{array}$ & 26.11 & .04 & .55 & .11 & 24.8 & $\begin{array}{r}.00 \\
0\end{array}$ & 1.74 & \multirow[t]{2}{*}{$\begin{array}{r}1.40- \\
2.56\end{array}$} \\
\hline & Constante & & & -1.04 & .24 & $\begin{array}{r}17.8 \\
9\end{array}$ & $\begin{array}{r}.00 \\
0\end{array}$ & .35 & \\
\hline \multirow[t]{2}{*}{ MFEx } & $\begin{array}{l}\text { Clasificados } \\
\text { correctamente: } \\
57.6 \%\end{array}$ & 15.06 & .25 & -.39 & .10 & $\begin{array}{r}14.6 \\
5\end{array}$ & $\begin{array}{r}.00 \\
0\end{array}$ & .67 & \multirow[t]{2}{*}{$.55-.76$} \\
\hline & Constante & & & 1.76 & .43 & $\begin{array}{r}16.7 \\
5 \\
\end{array}$ & $\begin{array}{r}.00 \\
0 \\
\end{array}$ & 5.85 & \\
\hline
\end{tabular}

Nota: MEC= Éxito en matemáticas atribuido a la capacidad; MEEs= Éxito en matemáticas atribuido al esfuerzo; $M E E x=$ Éxito en matemáticas atribuido a causas externas; MFC= Fracaso en matemáticas atribuido a la capacidad; MFEs $=$ Fracaso en matemáticas atribuido al esfuerzo; MFEx $=$ Fracaso en matemáticas atribuido a causas externas

\section{Modelo de regresión logística para la probabilidad de presentar alta ansiedad cognitiva en fun- ción de las atribuciones causales en matemáticas incluyendo todas las variables}

La Tabla 2 presenta el modelo de regresión logística para ansiedad cognitiva, tras incluir todas las variables de atribuciones causales en el área de matemáticas, los resultados muestran que la proporción de casos clasificados correctamente es del $64.3 \%\left(\chi^{2}=88.38 ; p=<.05\right)$. Los componentes del modelo expresados por la $O R$ permiten pronosticar una alta ansiedad cognitiva, siendo 1.70 veces mayor la probabilidad de manifestar ansiedad escolar por cada punto que se incrementa la puntuación en MFC= Fracaso en matemáticas atribuido a la capacidad. Por otro lado, resultó ser menor, concretamente .81 para $\mathrm{MEC}=$ Éxito en matemáticas atribuido a la capacidad y de .78 para MFEx= Fracaso en matemáticas atribuido a causas externas, por cada punto que se incrementaban las puntuaciones en dichas variables. 


\section{ATRIBUCIONES DEL ALUMNADO DE MATEMÁTICAS Y MANIFESTACIONES DE ANSIEDAD EN LA ESCUELA}

Tabla 2

Modelo de regresión logística para la probabilidad de presentar alta ansiedad cognitiva en función de las atribuciones en matemáticas incluyendo todas las variables

\begin{tabular}{|c|c|c|c|c|c|c|c|c|c|}
\hline Variable & & $\chi^{2}$ & $\mathrm{R}^{2}$ & B & E.T. & Wald & $p$ & $O R$ & I.C. $95 \%$ \\
\hline & $\begin{array}{l}\text { Clasificados } \\
\text { correctamente: } \\
64.3 \%\end{array}$ & 88.38 & .14 & & & & & & \\
\hline MEC & & & & -.20 & $\begin{array}{l}.0 \\
7\end{array}$ & 6.67 & $\begin{array}{r}.01 \\
0\end{array}$ & .81 & $.70-.95$ \\
\hline MFC & & & & .53 & $\begin{array}{c}.0 \\
9\end{array}$ & 31.57 & $\begin{array}{r}.00 \\
0\end{array}$ & $\begin{array}{r}1.7 \\
0\end{array}$ & $1.41-.2 .05$ \\
\hline MFEx & & & & -.24 & $\begin{array}{r}.1 \\
1\end{array}$ & 4.80 & $\begin{array}{r}.02 \\
8\end{array}$ & .78 & $.62-.97$ \\
\hline & Constante & & & .79 & $\begin{array}{r}.6 \\
1 \\
\end{array}$ & 1.65 & $\begin{array}{r}.19 \\
8 \\
\end{array}$ & $\begin{array}{r}2.2 \\
1 \\
\end{array}$ & \\
\hline
\end{tabular}

Nota $:$ MEC $=$ Éxito en matemáticas atribuido a la capacidad; MFC= Fracaso en matemáticas atribuido a la capacidad; $\mathrm{MFEx}=$ Fracaso en matemáticas atribuido a causas externas

\section{Regresión logística binaria para la probabilidad de presentar alta ansiedad conductual en fun- ción de las atribuciones causales en matemáticas}

Los modelos resultantes (véase la Tabla 3) permiten hacer una estimación correcta del 63.7\% de los casos $\left(\chi^{2}=65.72 ; p=<.05\right)$ para la variable MEC= Éxito en matemáticas atribuido a la capacidad; de un $61.2 \%$ de los casos $\left(\chi^{2}=34.77 ; p=<.05\right)$ correspondiente a la variable MEEs= Éxito en matemáticas atribuido al esfuerzo; de un $57.2 \%$ de los casos $\left(\chi^{2}=12.57 ; p=<.05\right)$ correspondiente a la variable MEEx= Éxito en matemáticas atribuido a causas externas; de un $64.6 \%$ de los casos $\left(\chi^{2}=70.05 ; p=<.05\right)$ correspondiente a la variable MFC= Fracaso en matemáticas atribuido a la capacidad; de $59,6 \%$ de los casos $\left(\chi^{2}=18.22 ; p=<.05\right)$ para la variable MFEs= Fracaso en matemáticas atribuido al esfuerzo y de un $57.5 \%$ de los casos $\left(\chi^{2}=20.99 ; p=<.05\right)$ para la variable MFEx= Fracaso en matemáticas atribuido a causas externas. El valor de ajuste de los modelos ( $R^{2}$ Nagelkerke) se situó entre 02 para el modelo MEEX=Éxito en matemáticas atribuido a causas externas y de .12 para la variable MFC= Fracaso en matemáticas atribuido a la capacidad.

Los valores de la odd ratio $(O R)$ fueron superiores a 1 indicando, por tanto, que a medida que aumenta la puntuación en las variables de Atribuciones Causales en Matemáticas aumenta la posibilidad de presentar altas puntuaciones en ansiedad conductual, obteniendo valores en MFC= Fracaso en matemáticas atribuido a la capacidad de 1.92 y en MFEs= Fracaso en matemáticas atribuido al esfuerzo de 1.59 veces mayor por cada punto que aumentan las puntuaciones respectivamente en las dimensiones de ansiedad conductual en función atribuciones causales en el área de Matemática. A su vez, también se obtuvieron valores de la $O R$ inferiores a 1, por lo que la probabilidad de presentar alta ansiedad cognitiva fue menor, concretamente de .58 para MEC= Éxito en matemáticas atribuido a la capacidad, de .59 para MEEs= Éxito en matemáticas atribuido al esfuerzo; de .73 para MEEX= Éxito en matemáticas atribuido a causas externas y de .62 para MFEx= Fracaso en matemáticas atribuido a causas externas, por cada punto que se incrementaban las puntuaciones en dichas variables. 
Tabla 3

Regresión logística binaria para la probabilidad de presentar alta ansiedad conductual en función de las atribuciones en matemáticas

\begin{tabular}{|c|c|c|c|c|c|c|c|c|c|}
\hline Variable & & $\chi^{2}$ & $\mathrm{R}^{2}$ & B & E.T. & Wald & $p$ & $O R$ & I.C. $95 \%$ \\
\hline \multirow[t]{2}{*}{ MEC } & $\begin{array}{l}\text { Clasificados } \\
\text { correctamente: } 63.7 \%\end{array}$ & 65.72 & $\begin{array}{r}.1 \\
1\end{array}$ & -.54 & .07 & $\begin{array}{r}59.3 \\
5\end{array}$ & $\begin{array}{r}.00 \\
0\end{array}$ & .58 & $.50-.66$ \\
\hline & Constante & & & 1.50 & .18 & $\begin{array}{r}64.7 \\
0\end{array}$ & $\begin{array}{r}.00 \\
0\end{array}$ & $\begin{array}{r}4.4 \\
9\end{array}$ & \\
\hline \multirow[t]{2}{*}{ MEEs } & $\begin{array}{l}\text { Clasificados } \\
\text { correctamente: } \quad 61.2 \\
\%\end{array}$ & 34.77 & .06 & -.52 & .09 & $\begin{array}{r}32.3 \\
5\end{array}$ & $\begin{array}{r}.00 \\
0\end{array}$ & .59 & $.49-.70$ \\
\hline & Constante & & & 1.58 & .25 & $\begin{array}{r}38.9 \\
5\end{array}$ & $\begin{array}{r}.00 \\
0\end{array}$ & $\begin{array}{r}4.8 \\
5\end{array}$ & \\
\hline \multirow[t]{2}{*}{ MEEx } & $\begin{array}{l}\text { Clasificados } \\
\text { correctamente: } 57.2 \%\end{array}$ & 12.57 & .02 & -.30 & .08 & $\begin{array}{r}12.3 \\
3\end{array}$ & $\begin{array}{r}.00 \\
0\end{array}$ & .73 & $.62-.87$ \\
\hline & Constante & & & 1.42 & .35 & $\begin{array}{r}16.3 \\
5\end{array}$ & $\begin{array}{r}.00 \\
0\end{array}$ & $\begin{array}{r}4.1 \\
7\end{array}$ & \\
\hline \multirow[t]{2}{*}{ MFC } & $\begin{array}{l}\text { Clasificados } \\
\text { correctamente: } 64.6 \%\end{array}$ & 70.05 & .12 & .65 & .08 & $\begin{array}{r}62.2 \\
8\end{array}$ & $\begin{array}{r}.00 \\
0\end{array}$ & $\begin{array}{r}1.9 \\
2\end{array}$ & $\begin{array}{r}1.63- \\
2.25\end{array}$ \\
\hline & Constante & & & -.87 & .15 & $\begin{array}{r}31.9 \\
0\end{array}$ & $\begin{array}{r}.00 \\
0\end{array}$ & .41 & \\
\hline \multirow[t]{2}{*}{ MFEs } & $\begin{array}{l}\text { Clasificados } \\
\text { correctamente: } 59.6 \%\end{array}$ & 18.22 & .03 & .46 & .11 & $\begin{array}{r}17.5 \\
8\end{array}$ & $\begin{array}{r}.00 \\
0\end{array}$ & $\begin{array}{r}1.5 \\
9\end{array}$ & $\begin{array}{r}1.28- \\
1.99\end{array}$ \\
\hline & Constante & & & -.80 & .25 & $\begin{array}{r}10.0 \\
6\end{array}$ & $\begin{array}{r}.00 \\
2\end{array}$ & .44 & \\
\hline MFEx & $\begin{array}{l}\text { Clasificados } \\
\text { correctamente: } 57.5 \% \\
\text { Constante }\end{array}$ & 20.99 & .04 & $\begin{array}{l}-.47 \\
2.17\end{array}$ & $\begin{array}{l}.11 \\
.44\end{array}$ & $\begin{array}{r}20.1 \\
6 \\
24.0 \\
8\end{array}$ & $\begin{array}{r}.00 \\
0 \\
.00 \\
0\end{array}$ & $\begin{array}{r}.62 \\
8.7 \\
7\end{array}$ & $.50-.76$ \\
\hline
\end{tabular}

Nota $:$ MEC = Éxito en matemáticas atribuido a la capacidad; MEEs= Éxito en matemáticas atribuido al esfuerzo; $\mathrm{MEEx}=$ Éxito en matemáticas atribuido a causas externas; $\mathrm{MFC}=$ Fracaso en matemáticas atribuido a la capacidad; MFEs $=$ Fracaso en matemáticas atribuido al esfuerzo; MFEx $=$ Fracaso en matemáticas atribuido a causas externas

\section{Modelo de regresión logística para la probabilidad de presentar alta ansiedad conductual en función de las atribuciones causales en matemáticas incluyendo todas las variables}

La Tabla 4 presenta el modelo de regresión logística para ansiedad conductual, tras incluir todas las variables de atribuciones causales en el área de matemáticas, los resultados muestran que la proporción de casos clasificados correctamente es del $66.1 \%\left(\chi^{2}=107.19 ; p=<.05\right)$. Los componentes del modelo expresados por la $O R$ permiten pronosticar una alta ansiedad conductual, siendo 1.44 veces mayor la probabilidad de manifestar ansiedad escolar por cada punto que se incrementa la puntuación en MFC= Fracaso en matemáticas atribuido a la capacidad. Por otro lado, resultó ser menor, concretamente .64 para MEC= Éxito en matemáticas atribuido a la capacidad y para MFEX= Fracaso en matemáticas atribuido a causas externas, por cada punto que se incrementaban las puntuaciones en dichas variables. 


\section{ATRIBUCIONES DEL ALUMNADO DE MATEMÁTICAS Y MANIFESTACIONES DE ANSIEDAD EN LA ESCUELA}

Tabla 4

Modelo de regresión logística para la probabilidad de presentar alta ansiedad conductual en función de las atribuciones en matemáticas incluyendo todas las variables

\begin{tabular}{|c|c|c|c|c|c|c|c|c|c|}
\hline Variable & & $\chi^{2}$ & $\mathrm{R}^{2}$ & B & E.T. & Wald & $p$ & $O R$ & I.C. $95 \%$ \\
\hline & $\begin{array}{l}\text { Clasificados } \\
\text { correctamente: } \\
66.1 \%\end{array}$ & 107.19 & .17 & & & & & & \\
\hline MEC & & & & -.43 & $\begin{array}{r}.0 \\
8\end{array}$ & $\begin{array}{r}28.0 \\
7\end{array}$ & .000 & .64 & $.55-.76$ \\
\hline MFC & & & & .37 & $\begin{array}{l}.0 \\
9\end{array}$ & $\begin{array}{r}15.0 \\
8\end{array}$ & .000 & 1.44 & $\begin{array}{l}1.20- \\
.1 .74\end{array}$ \\
\hline MFEx & & & & -.44 & $\begin{array}{r}.1 \\
1\end{array}$ & $\begin{array}{r}13.8 \\
8\end{array}$ & .000 & .64 & $.51-.81$ \\
\hline & Constante & & & 2.42 & $\begin{array}{r}.6 \\
4 \\
\end{array}$ & $\begin{array}{r}14.2 \\
9 \\
\end{array}$ & .000 & 11.34 & \\
\hline
\end{tabular}

Nota $:$ MEC $=$ Éxito en matemáticas atribuido a la capacidad; MFC $=$ Fracaso en matemáticas atribuido a la capacidad; MFEx $=$ Fracaso en matemáticas atribuido a causas externas

\section{Regresión logística binaria para la probabilidad de presentar alta ansiedad psicofisiológica en función de las atribuciones causales en matemáticas}

Los modelos resultantes (véase la Tabla 5) permiten hacer una estimación correcta del 59.6\% de los casos $\left(\chi^{2}=36.91 ; p=<.05\right)$ para la variable MEC= Éxito en matemáticas atribuido a la capacidad; de un $57.1 \%$ de los casos $\left(\chi^{2}=6.03 ; p=<.05\right)$ correspondiente a la variable MEEs= Éxito en matemáticas atribuido al esfuerzo; de un $54.9 \%$ de los casos $\left(\chi^{2}=4.43 ; p=<.05\right)$ correspondiente a la variable MEEX Éxito en matemáticas atribuido a causas externas; de un $62.4 \%$ de los casos $\left(\chi^{2}=61.46 ; p=<.05\right)$ correspondiente a la variable MFC= Fracaso en matemáticas atribuido a la capacidad; de $56,4 \%$ de los casos $\left(\chi^{2}=12.30 ; p=<.05\right)$ para la variable MFEs= Fracaso en matemáticas atribuido al esfuerzo y de un $53.8 \%$ de los casos $\left(\chi^{2}=5.92 ; p=<.05\right)$ para la variable MFEx= Fracaso en matemáticas atribuido a causas externas. El valor de ajuste de los modelos ( $R^{2}$ Nagelkerke) se situó entre .10 para la variable MFC= Fracaso en matemáticas atribuido a la capacidad y .01 para los modelos MEEs, MEE, y MFEx.

Los valores de la odd ratio $(O R)$ fueron superiores a 1 indicando, por tanto, que a medida que aumenta la puntuación en las variables de Atribuciones Causales en Matemáticas aumenta la posibilidad de presentar altas puntuaciones en ansiedad psicofisiológica, obteniendo valores en MFC= Fracaso en matemáticas atribuido a la capacidad de 1.83 y en MFEs= Fracaso en matemáticas atribuido al esfuerzo de 1.46 veces mayor por cada punto que aumentan las puntuaciones respectivamente en las dimensiones de ansiedad psicofisiológica en función atribuciones causales en el área de Matemática. A su vez, también se obtuvieron valores de la $O R$ inferiores a 1, por lo que la probabilidad de presentar alta ansiedad cognitiva fue menor, concretamente de .67 para MEC= Éxito en matemáticas atribuido a la capacidad, de .80 para MEES= Éxito en matemáticas atribuido al esfuerzo; de .83 para MEEX= Éxito en matemáticas atribuido a causas externas y de .77 para MFEX= Fracaso en matemáticas atribuido a causas externas, por cada punto que se incrementaban las puntuaciones en dichas variables. 
Tabla 5

Regresión logística binaria para la probabilidad de presentar alta ansiedad psicofisiológica en función de las atribuciones en matemáticas

\begin{tabular}{|c|c|c|c|c|c|c|c|c|c|}
\hline Variable & & $\chi^{2}$ & $\mathrm{R}^{2}$ & B & E.T. & Wald & $p$ & $O R$ & I.C. $95 \%$ \\
\hline \multirow[t]{2}{*}{ MEC } & $\begin{array}{l}\text { Clasificados } \\
\text { correctamente: } \\
59.6 \%\end{array}$ & 36.91 & .06 & -.39 & .06 & 34.83 & .000 & .67 & \multirow[t]{2}{*}{$.58-.76$} \\
\hline & Constante & & & 1.10 & .18 & 37.58 & .000 & 3.02 & \\
\hline \multirow[t]{2}{*}{ MEEs } & $\begin{array}{l}\text { Clasificados } \\
\text { correctamente: } \\
57.1 \%\end{array}$ & 6.03 & .01 & -.21 & .08 & 5.96 & .015 & .80 & \multirow[t]{2}{*}{$.68-.96$} \\
\hline & Constante & & & .70 & .24 & 8.64 & .003 & 2.02 & \\
\hline \multirow[t]{2}{*}{ MEEx } & $\begin{array}{l}\text { Clasificados } \\
\text { correctamente: } \\
54.9 \%\end{array}$ & 4.43 & .01 & -.18 & .08 & 4.40 & .036 & .83 & \multirow[t]{2}{*}{$.69-.98$} \\
\hline & Constante & & & .88 & .36 & 6.08 & .014 & 2.43 & \\
\hline \multirow[t]{2}{*}{ MFC } & $\begin{array}{l}\text { Clasificados } \\
\text { correctamente: } \\
62.4 \%\end{array}$ & 61.46 & .10 & .60 & .08 & 55.47 & .000 & 1.83 & \multirow[t]{2}{*}{$\begin{array}{r}1.56 \\
2.14\end{array}$} \\
\hline & Constante & & & -.81 & .14 & 30.52 & .000 & .44 & \\
\hline \multirow[t]{2}{*}{ MFEs } & $\begin{array}{l}\text { Clasificados } \\
\text { correctamente: } \\
56.4 \%\end{array}$ & 12.30 & .02 & .38 & .11 & 12.00 & .001 & 1.46 & \multirow[t]{2}{*}{$\begin{array}{r}1.18 \\
1.18\end{array}$} \\
\hline & Constante & & & -.67 & .24 & 7.39 & .007 & .51 & \\
\hline \multirow[t]{2}{*}{ MFEx } & $\begin{array}{l}\text { Clasificados } \\
\text { correctamente: } \\
53.8 \%\end{array}$ & 5.92 & .01 & -.25 & .10 & 5.86 & .015 & .77 & \multirow[t]{2}{*}{$.63-.95$} \\
\hline & Constante & & & 1.18 & .43 & 7.43 & .006 & 3.27 & \\
\hline
\end{tabular}

Nota: $\mathrm{MEC}=$ Éxito en matemáticas atribuido a la capacidad; MEEs= Éxito en matemáticas atribuido al esfuerzo; $\mathrm{MEEx}=$ Éxito en matemáticas atribuido a causas externas; $\mathrm{MFC}=$ Fracaso en matemáticas atribuido a la capacidad; MFEs $=$ Fracaso en matemáticas atribuido al esfuerzo; MFEx $=$ Fracaso en matemáticas atribuido a causas externas

\section{Modelo de regresión logística para la probabilidad de presentar alta ansiedad psicofisiológica en función de las atribuciones causales en matemáticas incluyendo todas las variables}

La Tabla 6 presenta el modelo de regresión logística para ansiedad psicofisiológica, tras incluir todas las variables de atribuciones causales en el área de matemáticas, los resultados muestran que la proporción de casos clasificados correctamente es del $63.6 \%\left(\chi^{2}=70.06 ; p=<.05\right)$. Los componentes del modelo expresados por la $O R$ permiten pronosticar una alta ansiedad psicofisiológica, siendo 1.46 veces mayor la probabilidad de manifestar ansiedad escolar por cada punto que se incrementa la puntuación en MFC= Fracaso en matemáticas atribuido a la capacidad y 1.64 veces menor la probabilidad de presentar alta ansiedad psicofisiológica por cada punto que aumentaba la puntuación MFEx= Fracaso en matemáticas atribuido a causas externas Por otro lado, resultó ser menor, concretamente .66 para MEC= Éxito en matemáticas atribuido a la capacidad por cada punto que se incrementaban las puntuaciones en esta variable. 


\section{ATRIBUCIONES DEL ALUMNADO DE MATEMÁTICAS Y MANIFESTACIONES DE ANSIEDAD EN LA ESCUELA}

Tabla 6

Modelo de regresión logística para la probabilidad de presentar alta puntuación en ansiedad psicofisiológica en función de las atribuciones en matemáticas incluyendo todas las variables

\begin{tabular}{|c|c|c|c|c|c|c|c|c|c|}
\hline Variable & & $\chi^{2}$ & $\mathrm{R}^{2}$ & B & E.T. & Wald & $p$ & $O R$ & I.C. $95 \%$ \\
\hline & $\begin{array}{l}\text { Clasificados } \\
\text { correctamente: } \\
63.6 \%\end{array}$ & 76,06 & $\begin{array}{l}.1 \\
3\end{array}$ & & & & & & \\
\hline MEC & & & &,- 40 & $\begin{array}{l}, 1 \\
0\end{array}$ & 13,92 & ,000 & ,66 &, $53-.82$ \\
\hline MFC & & & &, 37 & $\begin{array}{l}, 1 \\
3\end{array}$ & 7,67 & ,006 & 1,46 & $1,11-.1 .90$ \\
\hline \multirow[t]{2}{*}{ MFFx } & & & & ,49 & $\begin{array}{l}, 0 \\
9\end{array}$ & 29,65 &, 000 & 1,64 & $1,37-1.97$ \\
\hline & Constante & & &,- 65 & $\begin{array}{l}3 \\
4\end{array}$ & 3,63 & ,057 &, 52 & \\
\hline
\end{tabular}

Nota $:$ MEC $=$ Éxito en matemáticas atribuido a la capacidad; MFC $=$ Fracaso en matemáticas atribuido a la capacidad; $M F E x=$ Fracaso en matemáticas atribuido a causas externas

\section{DISCUSIÓN}

Los resultados obtenidos por los participantes de este estudio indican que a medida que aumenta la puntuación en atribuciones causales en matemáticas aumenta la posibilidad de presentar altas puntuaciones en ansiedad cognitiva, obteniendo valores altos en fracaso atribuido a la capacidad y al esfuerzo, por cada punto que aumentan las las dimensiones atribucionales. A su vez, también se obtuvo que la probabilidad de presentar alta ansiedad cognitiva fue menor para el éxito en matemáticas atribuido a la capacidad, al esfuerzo y a causas externas, y para fracaso en matemáticas atribuido a causas externas.

Por otro lado, a medida que aumenta la puntuación en las variables de atribuciones causales en matemáticas aumenta la posibilidad de presentar altas puntuaciones en ansiedad conductual, obteniendo valores mayores en fracaso atribuido a la capacidad y al esfuerzo. También se halló que la probabilidad de presentar alta ansiedad cognitiva fue menor para éxito atribuido a la capacidad, al esfuerzo y a causas externas.

Finalmente, se obtuvo que a medida que aumenta la puntuación en las variables de atribuciones causales aumenta la posibilidad de presentar alta ansiedad psicofisiológica, obteniendo valores mayores en fracaso atribuido a la capacidad y al esfuerzo. La probabilidad de presentar alta ansiedad psicofisiológica fue menor para el éxito atribuido a la capacidad, al esfuerzo y a causas externas y para fracaso en matemáticas atribuido a causas externas. Estos resultados son coincidentes con los obtenidos en la investigación sobre la regresión logística para la probabilidad de presentar alta ansiedad cognitiva y conductual, los mismos que permiten confirmar las tres hipótesis planteadas en base a trabajos previos (Cerezo y Casanova, 2004; Ferreira et al., 2002; Stipek y Gralinski, 1991).

\section{CONCLUSIONES}

Este trabajo permite concluir que resulta necesario redirigir las atribuciones causales del alumnado hacia un estilo adaptativo para evitar altos niveles de ansiedad. En este sentido, la evidencia de estudios experimentales hasta ahora sugiere que muchos procesos cognitivos se pueden entrenar mediante la autorregulación (Galla et al., 2019), desarrollando intervenciones específicas para el área de matemáticas (Fernández-Sogorb et al., 2020b).

\section{REFERENCIAS BIBLIOGRÁFICAS}

Cerezo, M. T. y Casanova, P. F. (2004). Gender differences in academic motivation of secondary school students. Revista Electrónica de Investigación Psicoeducativa, 2(1), 97-112.

Fernández-Sogorb, A., Inglés, C. J., Sanmartín, R., Gonzálvez, C., Vicent, M. y García-Fernández, J. M. (2018). 
Validation of the Visual Analogue Scale for Anxiety-Revised and school refusal across anxiety profiles. International Journal of Clinical and Health Psychology, 18(3), 264-272.

Fernández-Sogorb, A., Sanmartín, R., Vicent, M. y García-Fernández, J. M. (2020a). Latent profiles of anxious children and their differences in aggressive behavior. Sustainability, 12(15), 6199.

Fernández-Sogorb, A., Vicent, M., Gonzálvez, C., Sanmartín, R., Pérez-Sánchez, A. M. y García-Fernández, J. M. (2020b). Attributional style in mathematics across anxiety profiles in spanish children. Sustainability, 12(3), 1173.

Ferreira, M. C., Leal, E. M. A., Omar, A. G., Delgado, H. U., González, A. T., Silva, J. M. B. y de Cisne Figueiredo, M. D. C. (2002). Causal attribution to success and failure in school: a transcultural study Brazil-ArgentinMexico. Psicologia: Reflexão e Crítica, 15(3), 515-527.

Galla, B. M., Shulman, E. P., Plummer, B. D., Gardner, M., Hutt, S. J., Goyer, J. P., ... Duckworth, A. L. (2019). Why high school grades are better predictors of on-time college graduation than are admissions test scores: The roles of self-regulation and cognitive ability. American Educational Research Journal, 56(6), 2077-2115.

García-Fernández, J. M., Inglés, C. J., Martínez-Monteagudo, M. C., Marzo, J. C. y Estévez, E. (2011). Inventario de Ansiedad Escolar: validación en una muestra de estudiantes de Educación Secundaria. Psicothema, 23(2), 301-307.

Marsh, H. W. (1984). Relations among dimensions of self-attribution, dimensions of self-concept, and academic achievements. Journal of Educational Psychology, 76(6), 1291.

Martinko, M. J. y Mackey, J. D. (2019). Attribution theory: An introduction to the special issue. Journal of Organizational Behavior, 40(5), 523-527.

Stipek, D. J. y Gralinski, J. H. (1991). Gender differences in children's achievement-related beliefs and emotional responses to success and failure in mathematics. Journal of Educational Psychology, 83(3), 361-371. 
